\title{
CLIFFORD MODULE INVARIANTS OF SPIN BUNDLES
}

\author{
BY \\ JACQUES ALLARD ${ }^{1}$ AND ANTHONY BAHRI ${ }^{2}$
}

\begin{abstract}
In this paper, we study $\mathrm{KO}$-theory invariants of Spin bundles obtained by the $\alpha$-construction from Clifford module representations of the Spinor group. We begin by describing their elementary properties including various Whitney sum formulae and their relation with the $d$-invariant for vector bundles over spheres. We next observe an important difference between the two half-Spin representations and then proceed to investigate the fiber homotopy properties of the invariants. We conclude with some applications.
\end{abstract}

1. Introduction. Let $\xi$ be a principal Spin( $l$ )-bundle over a finite $\mathrm{CW}$-complex $X$ and $\rho$ one of the pure Spin representations of the spinor group $\operatorname{Spin}(l)$. Then, the correspondence $\xi \rightarrow \rho(\xi)$ is called a real, complex or quaternionic Spin operation according to whether $\rho$ is real, complex or quaternionic. The result $\rho(\xi)$ of the operation is an element of $K O(X), K(X)$ or $K S p(X)$ correspondingly. In this note, we describe properties of these operations and give some applications. We concentrate our attention on the real and quaternionic cases.

The paper is organized as follows. In $\$ 2$, we establish some notation to be used throughout the paper. $\S 3$ contains the definition of the Spin operations and $\S 4$ gives the basic properties. In $\S 5$, we describe the link between the Spin operations and the $d$-invariant. A consequence of this observation is that the class of Spin bundles on which the Spin operations are nontrivial is large. $\$ 6$ highlights a relationship between the value of certain Spin operations and the existence of complex structures on vector bundles. This result allows us to point out an important difference between the half-Spin representations of $\operatorname{Spin}(4 m)$. In $§ 7$, we discuss the relationship between the value of Spin operations and fiber homotopy triviality. Finally, in $\$ 8$ we give some applications. Spin operations appear to be most useful in studying stably trivial bundles. Our applications are related to such bundles. Among these is a theorem on the nonexistence of Samelson products and various nonsectioning results leading to an easy proof of a theorem of Barratt and Mahowald on the desuspension of Whitehead products.

The authors would like to acknowledge the help and advice of Professors K. Y. Lam and E. G. Rees. Much of the work presented here forms part of the authors' theses $[3,6]$, written under their direction.

\footnotetext{
Received by the editors May 15, 1981 and, in revised form, September 14, 1981.

1980 Mathematics Subject Classification. Primary 55R25, 15A66; Secondary 55S40, 55R50, 55R40.

'Partially supported by the National Sciences and Engineering Research Council of Canada, Grant \# A-4808.

${ }^{2}$ Partially supported by a National Science Foundation Grant.
} 
2. Notation. Throughout this paper, $X$ will denote a finite $\mathrm{CW}$-complex with base point. In general, $\xi^{l}$ will denote a principal Spin(l)-bundle over $X$. If $H^{l}\left(X ; Z_{2}\right) \cong 0$, then $\xi^{l}$ will denote either a principal Spin $(l)$-bundle over $X$ as above or a real vector bundle of dimension $l$ over $X$ whose second Stiefel-Whitney class is trivial and of which the first bundle is the unique Spin reduction. If $\eta^{m}$ is another such bundle, the sum bundle $\xi \oplus \eta$ is the bundle obtained by applying the continuous functor associated to the natural group homomorphism $\operatorname{Spin}(l) \times \operatorname{Spin}(m) \rightarrow \operatorname{Spin}(l+m)$.

The symbol $\Lambda$ will denote one of the finite dimensional associative division algebras over the real numbers, $R, C$ or $H$. The trivial $\Lambda$ line bundle will be denoted by $\varepsilon_{\Lambda}$. Furthermore, $K_{\Lambda}(X)$ will stand for $K O(X), K(X)$ or $K S p(X)$ according to the value of $\Lambda$. The $n$-sphere will be denoted $S^{n}$ and $\gamma_{\Lambda, n}$ will denote a generator of $\tilde{K}_{\Lambda}\left(S^{n}\right)$ if $\tilde{K}_{\Lambda}\left(S^{n}\right) \neq 0$.

Similarly, if $G$ is a compact Lie group, $R_{\Lambda}(G)$ will denote one of the representation rings or group of $G, R O(G), R(G)$ or $R S p(G)$. Following [2], the symbols $c, c^{\prime}, r$ and $q$ will denote the various changes of structure (complexification, etc.) for group representations, vector bundles and elements of $K_{\Lambda}(X)$ or $R_{\Lambda}(G)$.

Finally, given $\rho \in R_{\Lambda}(G)$ and a principal $G$-bundle $\zeta$ over $X, \alpha(\zeta, \rho)$ will denote the element of $K_{\Lambda}(X)$ obtained by the $\alpha$-construction. If $X \simeq \Sigma Y$ is a suspension, $\beta(\zeta ; \rho)$ will denote the element of $K_{\Lambda}^{-1}(Y)$ obtained by the $\beta$-construction.

3. Definition of the Spin operations. Let

$$
\Delta_{l}=\Delta_{C, l}: \operatorname{Spin}(l) \rightarrow U\left(2^{(l-1) / 2}\right) \text { for } l=1,3, \ldots
$$

and

$$
\Delta_{l}^{ \pm}=\Delta_{C, l}^{ \pm}: \operatorname{Spin}(l) \rightarrow U\left(2^{(l-2) / 2}\right) \quad \text { for } l=2,4, \ldots
$$

be the irreducible complex Spin representations of the Spinor group $\operatorname{Spin}(l)$ (see $[10,13, \S 9])$. The irreducible real and quaternionic Spin representations can be described by their image under the proper complexification map and are given in Table 1. (See $[9, \S 10]$ for details on how to make up such a list.)

TABLE 1. Irreducible real and quaternionic Spin representations.

\begin{tabular}{|c|c|c|c|c|c|c|c|c|c|}
\hline & $l \bmod 8$ & 0 & 1 & 2 & 3 & 4 & 5 & 6 & 7 \\
\hline \multirow{4}{*}{ Real } & $\theta$ & $\Delta_{R, l}^{ \pm}$ & $\Delta_{R, l}$ & $\Delta_{R, l}$ & $\Delta_{R, l}$ & $\Delta_{R, l}^{ \pm}$ & $\Delta_{R, l}$ & $\Delta_{R, l}$ & $\Delta_{R, l}$ \\
\cline { 2 - 9 } & $c(\theta)$ & $\Delta_{l}^{ \pm}$ & $\Delta_{l}$ & $\Delta_{l}^{+}+\Delta_{l}^{-}$ & $2 \Delta_{l}$ & $2 \Delta_{l}^{ \pm}$ & $2 \Delta_{l}$ & $\Delta_{l}^{+}+\Delta_{l}^{-}$ & $\Delta_{l}$ \\
\hline \multirow{4}{*}{ Quaternionic } & $\theta^{\prime}$ & $\Delta_{H, l}^{ \pm}$ & $\Delta_{H, l}$ & $\Delta_{H, l}$ & $\Delta_{H, l}$ & $\Delta_{H, l}^{ \pm}$ & $\Delta_{H, l}$ & $\Delta_{H, l}$ & $\Delta_{H, l}$ \\
\cline { 2 - 9 } & $c^{\prime}\left(\theta^{\prime}\right)$ & $2 \Delta_{l}^{ \pm}$ & $2 \Delta_{l}$ & $\Delta_{l}^{+}+\Delta_{l}^{-}$ & $\Delta_{l}$ & $\Delta_{l}^{ \pm}$ & $\Delta_{l}$ & $\Delta_{l}^{+}+\Delta_{l}^{-}$ & $2 \Delta_{l}$ \\
\hline
\end{tabular}

We now apply the $\alpha$-construction relative to $\xi$ to obtain elements of $\tilde{K}_{\Lambda}(X)$ as follows. 
If $\Lambda=C$, assume that $l \equiv 0$ (2). If $\Lambda=R$ or $H$, assume that $l \equiv 0$ (4). Then let

$$
\delta_{\Lambda}^{ \pm}(\xi)=\Delta_{\Lambda}^{ \pm}(\xi)-\left(\operatorname{dim}_{\Lambda} \Delta_{\Lambda, l}^{ \pm}\right) \varepsilon_{\Lambda} .
$$

For convenience, also define $\delta_{\Lambda}(\xi)=\delta_{\Lambda}^{+}(\xi)+\delta_{\Lambda}^{-}(\xi)$ and $\chi_{\text {Spin, } \Lambda}(\xi)=\delta_{\Lambda}^{+}(\xi)-\delta_{\Lambda}^{-}(\xi)$.

In the other cases $(\Lambda=C$ and $l \neq 0$ (2) or $\Lambda=R$ or $H$ and $l \neq 0$ (4)), let

$$
\delta_{\Lambda}(\xi)=\Delta_{\Lambda, l}(\xi)-\left(\operatorname{dim}_{\Lambda} \Delta_{\Lambda, l}\right) \varepsilon_{\Lambda} .
$$

The operators $\delta_{\Lambda}(-)$ and $\delta_{\Lambda}^{ \pm}(-)$are referred to as the real, complex or quaternionic Spin operations according to the value of $\Lambda$. In the complex case we often omit $\Lambda$.

The complex Spin operations were described in [4]. We therefore concentrate on the real and quaternionic cases.

REMARK (3.1). If $l \equiv 1(8)$, the Spin operation $\delta_{R}(-)$ is closely related to $\rho^{2}$, the 2nd cannibalistic class of Adams [1]. In fact,

$$
\delta_{R}(\xi)=\rho^{2}(\xi)+2^{(l-1) / 2} \varepsilon_{R} .
$$

See [9, Proposition 13.3], noting that Bott uses the notation $\theta_{2}$ for the class $\rho^{2}$.

4. Elementary properties of the Spin operations. The following theorem summarizes the basic properties of the real and quaternionic Spin operations. In particular, it includes formulae to compute the operations on sums of bundles.

Define $a_{\Lambda, l}=\operatorname{dim}_{\Lambda}\left(\Delta_{\Lambda, l}^{+}+\Delta_{\Lambda, l}^{-}\right)$for $l \equiv 0$ (4) and $a_{\Lambda, l}=\operatorname{dim}_{\Lambda} \Delta_{\Lambda, l}$ otherwise. Let $\phi(l)$ denote the number of integers $s$ such that $0<s \leqslant l$ and $s \equiv 0,1,2,4$ (8). Then, $a_{R, l}=2^{\phi(l-1)+1}$ for $l \equiv 0$ (4) and $a_{R, l}=2^{\phi(l-1)}$ otherwise. In all cases, $a_{H, l}=$ $2^{-3} a_{R, I+4}$.

THEOREM (4.1). Let $\xi$ and $\eta$ be principal $\operatorname{Spin}(l)$ - and $\operatorname{Spin}(m)$-bundles over a finite connected $C W$-complex $X$. Unless otherwise stated, assume that $m \equiv 0$ (4). Let $\Lambda=R$ or $H$. Then:

(i) $\delta_{\Lambda}(\xi)$ and $\delta_{\Lambda}^{ \pm}(\eta)$ depend only on the isomorphism class of $\xi$ and $\eta$ respectively.

(ii) If $Y$ is another finite connected $C W$-complex and $f: Y \rightarrow X$ is a continuous map, then $\delta_{\Lambda} f^{*}(\xi)=f^{!} \delta_{\Lambda}(\xi)$ and $\delta_{\Lambda}^{ \pm} f^{*}(\eta)=f^{!} \delta_{\Lambda}^{ \pm}(\eta)$.

(iii) If $\xi($ resp. $\eta)$ is trivial, then $\delta_{\Lambda}(\xi)=0\left(\right.$ resp. $\left.\delta_{\Lambda}^{ \pm}(\eta)=0\right)$.

(iv) $c \delta_{R}(\xi)=k \delta(\xi)$ and $c \delta_{R}^{ \pm}(\eta)=k \delta^{ \pm}(\eta)$ where $k=2$ if $l \equiv 3,4,5$ (8) and $k=1$ otherwise.

(v) $c^{\prime} \delta_{H}(\xi)=k \delta(\xi)$ and $c^{\prime} \delta_{H}^{ \pm}(\eta)=k \delta^{ \pm}(\eta)$ where $k=2$ if $l \equiv 0,1,7$ (8) and $k=1$ otherwise.

(vi) For $l, m=1,2,3, \ldots$,

$$
\delta_{\Lambda}^{ \pm}(\xi \oplus \eta) \text { if } l+m \equiv 0(4) \quad \text { or } \quad \delta_{\Lambda}(\xi \oplus \eta) \text { if } l+m \neq 0(4)
$$

is given by Table 2 where in that table

$$
\begin{gathered}
A\left(\Lambda^{\prime}, \Lambda^{\prime \prime}\right)=\delta_{\Lambda^{\prime}}^{ \pm}(\xi) \cdot \delta_{\Lambda^{\prime \prime}}^{+}(\eta)+\delta_{\Lambda^{\prime}}^{\mp}(\xi) \cdot \delta_{\Lambda^{\prime \prime}}^{-}(\eta) \\
+\frac{1}{2} a_{\Lambda^{\prime}, l} \varepsilon_{\Lambda^{\prime}} \cdot \delta_{\Lambda^{\prime \prime}}(\eta)+\frac{1}{2} a_{\Lambda^{\prime \prime}, m^{\prime}} \delta_{\Lambda^{\prime}}(\xi) \cdot \varepsilon_{\Lambda^{\prime \prime}}, \\
B\left(\Lambda^{\prime}, \Lambda^{\prime \prime}\right)=\delta_{\Lambda^{\prime}}(\xi) \cdot \delta_{\Lambda^{\prime \prime}}(\eta)+a_{\Lambda^{\prime}, l} \varepsilon_{\Lambda^{\prime}} \cdot \delta_{\Lambda^{\prime \prime}}(\eta)+a_{\Lambda^{\prime \prime}, m} \delta_{\Lambda^{\prime}}(\xi) \cdot \varepsilon_{\Lambda^{\prime \prime}}, \\
C=\delta_{C}^{ \pm}(\xi) \cdot \delta_{C}^{+}(\eta)+2^{(l-2) / 2} \delta_{C}^{+}(\eta)+2^{(m-2) / 2} \delta_{C}^{ \pm}(\xi) .
\end{gathered}
$$


TABLE 2. Product formulae for real (top) and quaternionic (bottom) Spin Operations. (In each case, only a part of the table is given. The isomorphism $\xi \oplus \eta \cong \eta \oplus \xi$ makes the other part unnecessary.)

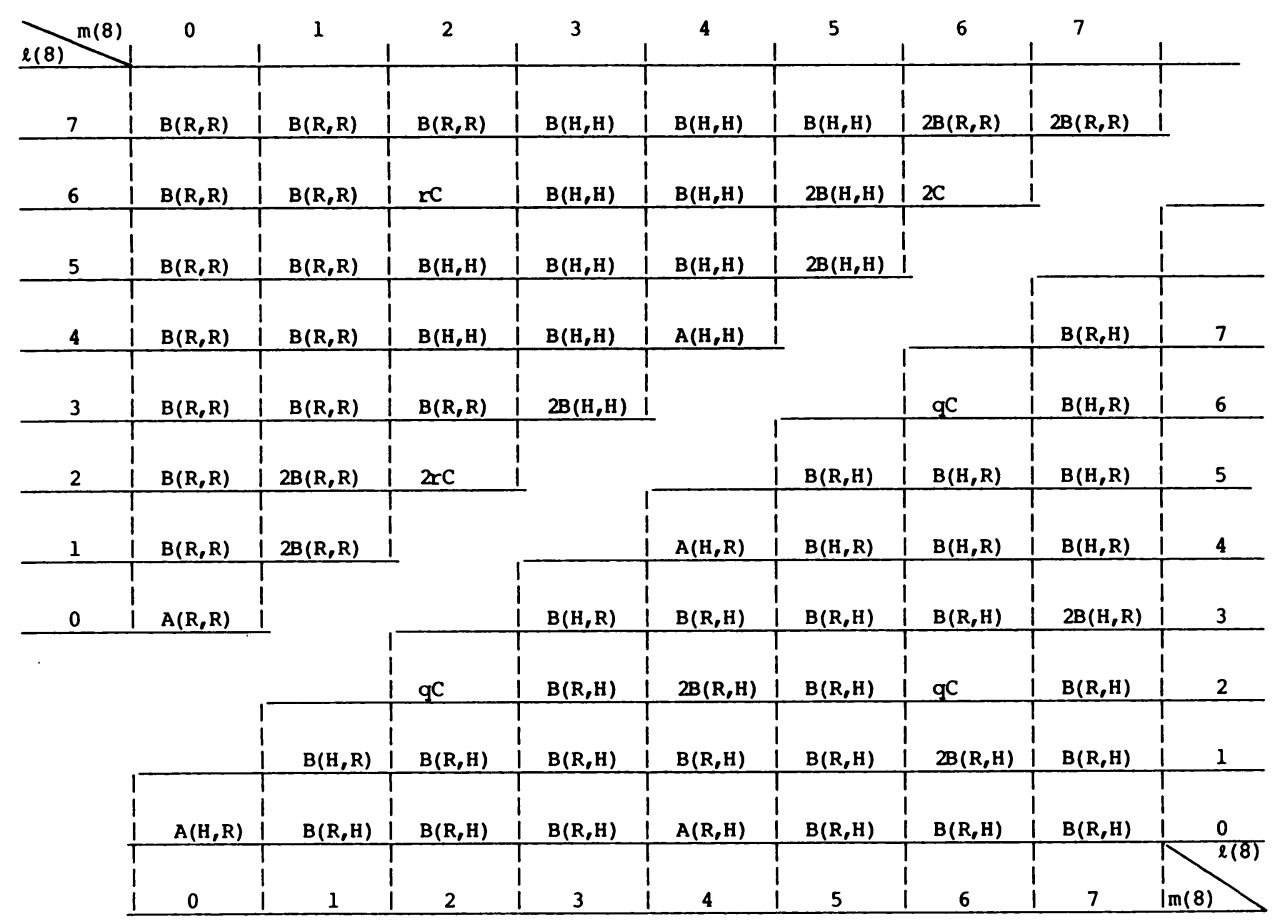

Proof. The first statements are immediate consequences of the properties of the $\alpha$-construction and the definitions. The proof of the last statement reduces to a standard character computation in the following way. Recall that $K O(X) \oplus K \operatorname{Sp}(X)$ and $R O(G) \oplus R S p(G)$ are $Z_{2}$-graded rings in a natural way. Moreover, there are natural $Z_{2}$-graded ring structures on $K U(X) \oplus K U(X)$ and $R(G) \oplus R(G)$ such that the morphisms represented in the following diagram are $Z_{2}$-graded ring homomorphisms (compare [15]):

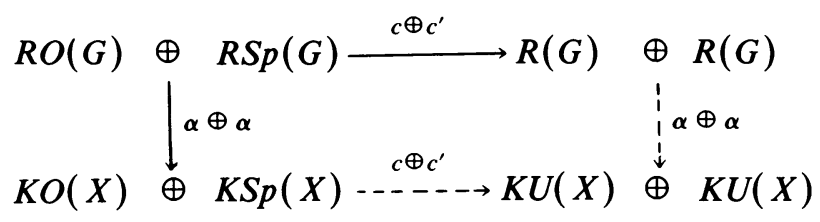

Here, the $\alpha$-construction is relative to some fixed principal $G$-bundle over $X$. The top horizontal arrow represents a monomorphism. The dotted arrows are not necessary for our purposes, but it is a fact that the square is commutative. Furthermore, this diagram is natural in various ways. In particular, if $G^{\prime}$ is another compact Lie group 
and $b: G \rightarrow G^{\prime}$ is a group homomorphism, the diagram is natural relative to $b$. Now let $G=\operatorname{Spin}(l) \times \operatorname{Spin}(m)$ and $G^{\prime}=\operatorname{Spin}(l+m)$. Furthermore, let $b: G \rightarrow G^{\prime}$ be the homomorphism covering the natural inclusion $S O(l) \times S O(m) \rightarrow S O(l+m)$. If $\Lambda=R$ or $H$ and $\rho \in R_{\Lambda}\left(G^{\prime}\right) \subset R O(G) \oplus R S p(G)$ is one of the Spin representations, then $b^{*} \rho$ can be computed by evaluating $\left(c \oplus c^{\prime}\right) b^{*} \rho=b^{*}\left(c \oplus c^{\prime}\right) \rho \in R(G) \oplus$ $R(G)$. This, however, is a straightforward character computation in $R(G) \oplus R(G)$. The last statement of the theorem then follows from this computation and easy naturality arguments.

REMARK (4.2). Formulae to compute the Spin operations on a product bundle over a product space follow immediately from the last statement of the theorem.

5. Relation with the $d$-invariant. In this section, we suppose that $X \simeq \Sigma Y$ is the suspension of a finite CW-complex $Y$. We also assume that $l \equiv 0 \bmod 2$ if $\Lambda=C$ and that $l \equiv 0(4)$ if $\Lambda=R$ or $H$.

Let $c_{\xi}: Y \rightarrow \operatorname{Spin}(l)$ denote the characteristic map of $\xi$. If $\rho \in R_{\Lambda}(\operatorname{Spin}(l))$ has dimension 0 , then $\alpha(\xi ; \rho) \in \tilde{K}_{\Lambda}(X)$ and $\beta(\xi ; \rho) \in \tilde{K}_{\Lambda}^{-1}(Y)$. Moreover, $\alpha(\xi ; \rho)=$ $\Sigma \beta(\xi ; \rho)$ where $\Sigma$ denotes the suspension homomorphism.

Now let $\rho=\Delta_{\Lambda, l}^{+}-\Delta_{\Lambda, l}^{-}$. By definition $\chi_{\text {Spin, } \Lambda}(\xi)=\alpha(\xi ; \rho)$. Consider the following diagram where $O_{\Lambda}(*)$ is the suitable group of matrices and $O_{\Lambda}$ is the corresponding stable object.

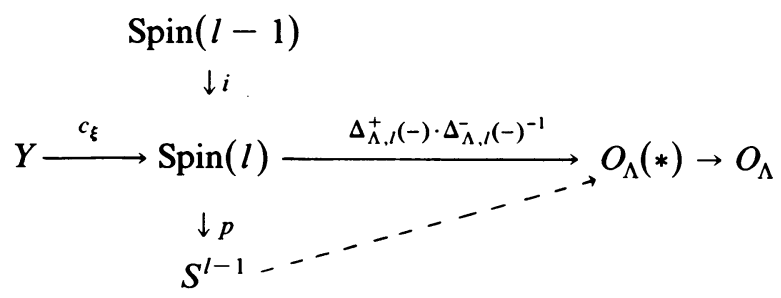

The composition $Y \rightarrow O_{\Lambda}$ is an explicit representative of $\beta(\xi ; \rho)$. Furthermore, since $\Delta_{\Lambda, l}^{+} \cdot i=\Delta_{\Lambda, l}^{-} \cdot i$ the map $\operatorname{Spin}(\rho) \rightarrow O_{\Lambda}(*)$ induces a map on the coset space $S^{l-1}$ as shown. The composition $S^{l-1} \rightarrow O_{\Lambda}$ represents a generator $\Sigma^{-1} \gamma_{\Lambda, l}$ of $\tilde{K}_{\Lambda}^{-1}\left(S^{l-1}\right)$. (To see this, replace $Y$ with $S^{l-1}$ and $c_{\xi}$ with $c_{\tau^{\prime}}$, the characteristic map of the tangent bundle of $S^{\prime}$. The results of [5] show that the horizontal composition is $2 \Sigma^{-1} \gamma_{\Lambda, l}$. Since $l$ is even, $p \cdot c_{\tau^{\prime}}=2 \in \pi_{l-1} S^{l-1}$ and so the dotted arrow must represent $\Sigma^{-1} \gamma_{\Lambda, l}$ ) We therefore have

$$
\chi_{\operatorname{Spin}, \Lambda}(\xi)=\alpha(\xi ; \rho)=\Sigma \beta(\xi ; \rho)=\Sigma c_{\xi}^{*} p^{*} \Sigma^{-1} \gamma_{\Lambda, l}
$$

Thus

$$
\chi_{\mathrm{Spin}, \Lambda}(\xi)=c_{\xi}^{*} p^{*} \gamma_{\Lambda, l}
$$

If $X=S^{l+r+1}$ and $\Lambda=R$, this result implies immediately the following theorem.

THEOREM (5.2). Let $\xi$ be a principal $\operatorname{Spin}(l)$-bundle over the sphere $S^{l+r+1}$ with characteristic map $c_{\xi} \in \pi_{l+r}(\operatorname{Spin}(l))$. Let $p: \operatorname{Spin}(l) \rightarrow S^{l-1}$ be the natural projection. Assume that $l \equiv 0$ (4). Then $\chi_{\text {Spin, } R}(\xi) \neq 0$ if and only if $d\left(p \cdot c_{\xi}\right) \neq 0$. 
REMARK (5.3). The work of Berrick [8] and of Mukai [14] shows that the class of maps $c: S^{l+r} \rightarrow \operatorname{Spin}(l)$ such that $d(p \cdot c) \neq 0$ is large.

6. Difference between $\delta_{\Lambda}^{+}$and $\delta_{\Lambda}^{-}$. In this section, we assume that $\Lambda=R$ or $H$ and that $l \equiv 0$ (4). We will highlight an important difference between $\delta_{\Lambda}^{+}$and $\delta_{\Lambda}^{-}$as invariants of real vector bundles. Let $l=4 k$. Recall that

$$
R S U(2 k) \cong Z\left[\lambda_{1}, \lambda_{2}, \ldots, \lambda_{2 k-1}\right]
$$

where $\lambda_{i}$ is the $i$ th exterior power of the regular representation of $S U(2 k)$. We have the following.

THEOREM (6.1). Let $X$ be a simply connected finite $C W$-complex and let $\xi^{4 k}$ be a real vector bundle over $X$ such that $w_{2}(\xi)=0$. Assume that $\xi \cong r \xi_{C}$ for some complex vector bundle $\xi_{C}$. Then:

(i) if $k \equiv 0(2), \delta_{R}^{-}(\xi)=r \alpha\left(\xi_{C} ; \sum_{i=0}^{(k-2) / 2} \lambda_{2 i+1}-2^{2 k-2}\right)$,

(ii) if $k \equiv 1(2), \delta_{H}^{+}(\xi)=q \alpha\left(\xi_{C} ; \sum_{i=0}^{(k-1) / 2} \lambda_{2 i}-2^{2 k-2}\right)$.

Proof. Let $j: S U(2 k) \rightarrow S O(4 k)$ be the natural inclusion and let $\bar{j}: S U(2 k) \rightarrow$ $\operatorname{Spin}(4 k)$ its unique lifting. A straightforward character computation shows that:

(i) if $k \equiv 0(2), j^{*}\left(\Delta_{R, 4 k}^{-}\right)=r\left(\sum_{i=0}^{(k-2) / 2} \lambda_{2 i+1}\right)$,

(ii) if $k \equiv 1(2), \bar{j}^{*}\left(\Delta_{H, 4 k}^{+}\right)=q\left(\sum_{i=0}^{(k-1) / 2} \lambda_{2 i}\right)$.

The theorem now follows by using the naturality of the $\alpha$-construction and the fact that the $\alpha$-construction commutes with $q$ and $r$.

The following corollary of Theorem (6.1) is immediate.

Corollary (6.2). Let $X$ and $\xi=r \xi_{C}$ be as in Theorem (6.1). Assume that $\tilde{K}(X)=0$. Then:

(i) if $k \equiv 0(2), \delta_{R}^{-}(\xi)=0$;

(ii) if $k \equiv 1(2), \delta_{H}^{+}(\xi)=0$.

7. Fiber homotopy and $Z_{2}$-equivariant fiber homotopy type. We shall now discuss the relationship between the $S$ pin operations, fiber homotopy type and $Z_{2}$-equivariant fiber homotopy type. For $l \equiv 1(8)$, Remark (2.1) and the work of Adams imply that, if $\xi$ is fiber homotopy trivial, there is an element $x \in \widetilde{K O}(X)$ such that $1+x$ is invertible and

$$
\delta_{R}(\xi)=\frac{2^{l / 2} \psi^{2}(1+x)}{1+x}
$$

(See $[9, \S 13]$.$) For l \equiv 0(8)$, we have the following

THEOREM (7.1). Let $\xi$ be a principal Spin(l)-bundle over a finite simply connected $C W$-complex $X$. Assume that $l \equiv 0(8)$. Then

$$
\delta_{R}^{+}(\xi)=0=\delta_{R}^{-}(\xi)
$$

if one of the following sets of conditions is satisfied.

(i) $K O(X)$ is torsion free and $\xi$ is stably trivial and fiber homotopically trivial;

(ii) $X=S^{r}$ where $r \equiv 1,2(8)$ and $r \leqslant 2 l-7$, and $\xi$ is fiber homotopically trivial;

(iii) $X=S^{r}$ where $r \equiv 1$ (8) and $\xi$ is $Z_{2}$-equivariantly fiber homotopically trivial. 
Proof. (i) To prove the theorem with the first set of conditions, let $S(\xi)$ be the sphere bundle associated to $\xi$ and let $\pi: S(\xi) \rightarrow X$ be the projection. Consider the following long exact sequence

$$
\leftarrow K O^{p-l+1}(X) \leftarrow K O^{p}(S(\xi)) \stackrel{\pi^{!}}{\leftarrow} K O^{p}(X) \stackrel{\phi}{\leftarrow} K O^{p-l}(X) \leftarrow
$$

where $\phi$ is multiplication by $\chi_{\text {Spin, } R}(\xi)=\delta_{R}^{+}(\xi)-\delta_{R}^{-}(\xi)[9$, Theorem 15.1]. Since $\xi$ is fiber homotopically trivial, it admits a homotopy section. Therefore $\pi^{!}$is a monomorphism and $\phi=0$. We deduce that

$$
\chi_{\text {Spin }, R}(\xi)=\delta_{R}(\xi)^{+}-\delta_{R}(\xi)^{-}=0 .
$$

Now, assume that $\xi$ is stably trivial of type $(n, k)$, that is $\xi \oplus k \varepsilon_{R} \cong n \varepsilon_{R}$. Applying the formulae of Theorem 4.1 we obtain

$$
a_{R, k}\left(\delta_{R}^{+}(\xi)+\delta_{R}^{-}(\xi)\right)=\delta_{R}\left(\xi \oplus k \varepsilon_{R}\right)=0 .
$$

Since $K O(X)$ is torsion-free, we deduce that

$$
\delta_{R}^{+}(\xi)+\delta_{R}^{-}(\xi)=0 .
$$

The desired conclusion follows immediately from (7.2) and (7.3).

(ii) We now prove the theorem with the second set of conditions. An easy argument based on the isomorphism

$$
\pi_{r-1}(S O(l), S O(l-k)) \cong \pi_{r+l-k-1}\left(\Omega^{k} S^{l}, S^{l-k}\right)
$$

valid for $r \leqslant 2(l-k)-1$ [11, Theorem 14.4] shows that, if $J(\xi)=0 \in \pi_{r+l-1}\left(S^{l}\right)$, then $c_{\xi} \in \pi_{r-1}(S O(l))$ is the image of an element $c_{\xi^{\prime}} \in \pi_{r-1}(S O(l-k))$. Therefore, $\xi \cong \xi^{\prime} \oplus k \varepsilon_{R}$. Taking $k=3$ and applying the formulae of Theorem (4.1) we obtain

$$
\delta_{R}^{ \pm}(\xi)=\delta_{R}^{ \pm}\left(\xi^{\prime} \oplus 3 \varepsilon_{R}\right)=\delta_{H}(\xi) \cdot \varepsilon_{H}=0 \in \widetilde{K O}\left(S^{r}\right) \text {. }
$$

(iii) Finally, we will assume the third set of conditions. Using the same argument as in part (i) above, we deduce that (7.2) holds in this case also. Let $\eta_{\xi}$ be the canonical line bundle over $R P(\xi)$, the projective bundle of $\xi$ and let $\bar{\pi}: R P(\xi) \rightarrow S^{r}$ be the projection. The following formula (see $[9, \S 18])$ relates $\delta_{R}^{+}(\xi)$ and $\delta_{R}^{-}(\xi)$ in $K O(R P(\xi))$ :

$$
\bar{\pi} ! \delta_{R}^{+}(\xi) \otimes \eta_{\xi}=\bar{\pi} ! \delta_{R}^{-}(\xi)
$$

Using (7.2), we deduce that

$$
\bar{\pi}^{!} \delta_{R}^{+}(\xi) \otimes\left(\eta_{\xi}-1\right)=0
$$

Now consider the following composition where the first map is the inclusion and the second is the fiber homotopy equivalence given by the $Z_{2}$-equivariant homotopy equivalence:

$$
S^{r} \times S^{1} \stackrel{i}{\rightarrow} S^{r} \times R P^{1-1} \stackrel{\phi}{\rightarrow} R P(\xi) .
$$

If $\delta_{R}^{+}(\xi)=\delta_{R}^{-}(\xi) \neq 0 \in \widetilde{K O}\left(S^{r}\right)$, it follows that $i^{!} \phi^{!}\left(\bar{\pi}^{!} \delta_{R}^{+}(\xi) \otimes\left(\eta_{\xi}-1\right)\right)$ is the product of the generators of $\widetilde{K O}\left(S^{1}\right)$ and $\widetilde{K O}\left(S^{r}\right)$. However, this product is nonzero, contradicting (7.5). This concludes the proof. 
REMARK (7.6). If $r \equiv 1$ (8), part (ii) of (7.1) still holds if $r \leqslant 2 l-5$. The proof is similar but uses the fact that $\widetilde{K U}\left(S^{8 k+1}\right)=0$.

REMARK (7.7). Similar results hold for $l \equiv 4(8)$ with $K S p(X)$ replacing $K O(X)$ at the proper places.

8. Applications. We now give a number of applications of the Spin operations. Some proofs are rather sketchy. Further details can be found in [ 3 and 6].

Before starting, we should recall the results of [5] concerning the tangent bundle $\tau^{l}$ of the $l$-sphere $S^{l}$. Stated in our vocabulary, they are

$$
\begin{array}{lll}
\delta_{\Lambda}^{ \pm}\left(\tau^{l}\right)= \pm \gamma_{\Lambda, l} & \text { for } l \equiv 0,4(8) & \text { and } \\
& \text { or } l \equiv 0(2) & \text { and } \Lambda=C, H \\
\delta_{\Lambda}\left(\tau^{l}\right)=\gamma_{\Lambda, l} & \text { for } l \equiv 1,2(8) & \text { and } \Lambda=R \\
& \text { or } l \equiv 5,6(8) & \text { and } \Lambda=H .
\end{array}
$$

(a) Subbundles of $\tau^{4 k+1}$. If $4 k+1 \geqslant 5$ there are two nonisomorphic vector bundles, $\zeta_{1}^{4 k}$ and $\zeta_{2}^{4 k}$, over $S^{4 k+1}$ satisfying the condition

$$
\zeta_{i} \oplus \varepsilon_{R}=\tau^{4 k+1},
$$

moreover $\zeta_{1}+\zeta_{2}=\tau^{4 k} \Sigma \eta$ and $p^{*}\left(\zeta_{i}\right)=\eta$ where $\eta$ is the generator of $\pi_{4 k} S^{4 k-1}$ and $p^{*}\left(\zeta_{i}\right)$ is the characteristic map of $\zeta_{i}$ composed with the projection $p: \operatorname{Spin}(4 k) \rightarrow$ $S^{4 k-1}$. One of these bundles, say $\zeta_{1}$, admits a complex structure and the other, $\zeta_{2}$, does not. We have the following proposition.

Proposition (8.2). Let $\zeta_{1}^{4 k}$ and $\zeta_{2}^{4 k}$ be as above. Then:

(i) if $k \equiv 0(2), \delta_{R}^{+}\left(\zeta_{1}\right)=\delta_{R}^{-}\left(\zeta_{2}\right) \neq 0=\delta_{R}^{-}\left(\zeta_{1}\right)=\delta_{R}^{+}\left(\zeta_{2}\right)$,

(ii) if $k \equiv 1(2), \delta_{H}^{-}\left(\zeta_{1}\right)=\delta_{H}^{+}\left(\zeta_{2}\right) \neq 0=\delta_{H}^{+}\left(\zeta_{1}\right)=\delta_{H}^{-}\left(\zeta_{2}\right)$.

Proof. Suppose that $k \equiv 0$ (2). Since the $d$-invariant of $\eta$ is not zero, we have by Theorem (3.2) that

$$
\delta_{R}^{+}\left(\zeta_{1}\right)-\delta_{R}^{-}\left(\zeta_{1}\right) \neq 0 \in K O\left(S^{4 k+1}\right)
$$

and so by part (i) of Corollary (6.2) $\delta_{R}^{+}\left(\zeta_{1}\right) \neq 0$ and $\delta_{R}^{-}\left(\zeta_{1}\right)=0$. For $\zeta_{2}$ we have

$$
\delta_{R}^{+}\left(\zeta_{1}+\zeta_{2}\right)=\delta_{R}^{+}\left(\zeta_{1}\right)+\delta_{R}^{+}\left(\zeta_{2}\right)=\delta_{R}^{+}\left(\tau^{4 k} \Sigma \eta\right)
$$

which is nonzero in $K O\left(S^{4 k+1}\right)$ by (8.1). So $\delta_{R}^{+}\left(\zeta_{2}\right)=0$ and similarly, $\delta_{R}^{-}\left(\zeta_{2}\right) \neq 0$. The proof for $k \equiv 1$ (2) is the same except that we use part (ii) of Corollary (6.2).

COROllary (8.3). Let $\zeta^{4 s}=\zeta_{1}^{4 s}$ or $\zeta_{2}^{4 s}$ be one of the bundles above. Assume that $k, l$ and $m \geqslant 1$. Then, the vector bundle $\zeta^{4 l} \times \zeta^{4 k} \times \tau^{4 m}$ over $S^{4 l+1} \times S^{4 k+1} \times S^{4 m}$ admits exactly one section.

Proof. Assume that $k \equiv l \equiv 0$ (2) and $m \equiv 1$ (2). The other cases are similar. Let $\xi=\zeta^{4 l} \times \zeta^{4 k} \times \tau^{4 m}$. Using (8.1) and (8.2), one easily obtains

$$
\delta_{R}^{+}\left(\zeta^{4 k} \times \zeta^{4 l} \times \tau^{4 m}\right)=\gamma_{R, 4 k+1} \otimes \gamma_{R, 4 k+1} \otimes \gamma_{R, 4 m}
$$

in $\widetilde{K O}\left(S^{4 k+1} \times S^{4 l+1} \times S^{4 m}\right)$. Therefore, $\xi$ is not trivial. Now assume that $\xi=\xi^{\prime}+$ $2 \varepsilon_{R}$ for some bundle $\xi^{\prime}$ over $S^{4 k+1} \times S^{4 l+1} \times S^{4 m}$. Using again the formulae of Theorem (4.1), a contradiction with (8.4) is obtained. Finally, it is easy to show that $\xi$ admits at least one section by usual means. 
(b) Detecting homotopy elements. Recall [13] that

$$
\pi_{4 k}(\operatorname{Spin}(4 k)) \cong \begin{cases}Z_{2}+Z_{2}+Z_{2} & \text { for } k \equiv 0(2), \\ Z_{2}+Z_{2} & \text { for } k \equiv 1(2) .\end{cases}
$$

Furthermore, the generators can be taken to be $c_{\eta^{*} \tau^{4 k}}, c_{\zeta_{1}}$ and, for $k$ even, a certain stable element $\alpha$ coming from $\pi_{4 k} \operatorname{Spin}(4 k-1)$. Consider the case $k \equiv 0$ (2). Define a map $C: \pi_{4 k}(\operatorname{Spin}(4 k)) \rightarrow\left(K O\left(S^{4 k+1}\right)\right)^{3}$ by

$$
\dot{C}\left(c_{\xi}\right)=\left(f_{\xi}^{\prime} \gamma, \delta_{R}^{+}(\xi), \delta_{R}^{-}(\xi)\right)
$$

where $c_{\xi}$ is the characteristic map of a bundle $\xi, f_{\xi}$ is the classifying map of $\xi$ and $\gamma \in \widehat{K O}(B \operatorname{Spin}(4 k))$ is the class of the composition $B \operatorname{Spin}(4 k) \rightarrow B S O(4 k) \rightarrow B O$.

For $k \equiv 1(2)$, define $C: \pi_{4 k}(\operatorname{Spin}(4 k)) \rightarrow\left(K O\left(S^{4 k+1}\right)\right)^{2}$ by

$$
C\left(c_{\xi}\right)=\left(\delta_{H}^{+}(\xi), \delta_{H}^{-}(\xi)\right) .
$$

Proposition (8.5). The maps $C$ are group isomorphisms.

Proof. This is checked by computing explicitly the values of $C$ with the help of (8.1) and (8.2).

This result is used in the following.

(c) Nonexistence of Samelson products. We now prove the following theorem.

THEOREM (8.6). Let $\mu_{8 r+1} \in \pi_{4 k+8 r+1}\left(S^{4 k}\right)$ be an element with nonzero d-invariant. Then the image of the map

$$
\mu_{8 r+1}^{\#}: \pi_{4 k}(\operatorname{Spin}(4 k)) \rightarrow \pi_{4 k+8 r+1}(\operatorname{Spin}(4 k))
$$

does not contain nontrivial Samelson products. In particular, $\pi_{4 k}(\operatorname{Spin}(4 k))$ and $\pi_{4 k+1}(\operatorname{Spin}(4 k))$ do not contain nontrivial Samelson products.

Proof. If $c_{\xi^{\prime}} \in \pi_{p-1}(\operatorname{Spin}(l))$ and $c_{\xi^{\prime \prime}} \in \pi_{q-1}(\operatorname{Spin}(l))$, the Samelson product $\left\langle c_{\xi^{\prime}}, c_{\xi^{\prime \prime}}\right\rangle$ of $c_{\xi^{\prime}}$ and $c_{\xi^{\prime \prime}}$ is the class $c_{\xi} \in \pi_{p+q-2} \operatorname{Spin}(l)$ such that $f_{\xi}= \pm\left[f_{\xi^{\prime}}, f_{\xi^{\prime \prime}}\right]$. Since $f_{\xi}$ is a Whitehead product, $f_{\xi}$ is trivial in any cohomology theory. In particular, if $c_{\xi} \in \pi_{4 k}(\operatorname{Spin}(4 k))$ and $k$ is even,

$$
C\left(c_{\xi}\right)=\left(f_{\xi}^{\prime} \gamma, f_{\xi}^{!} \delta_{R}^{+}\left(\gamma_{4 k}\right), f_{\xi}^{!} \delta_{R}^{+}\left(\gamma_{4 k}\right)\right)
$$

where $\gamma_{4 k}$ is the universal bundle over $B \operatorname{Spin}(4 k)$. By Proposition (8.5), $C\left(c_{\xi}\right) \neq 0$. Therefore, $c_{\xi}$ cannot be a Samelson product in a nontrivial way. Similarly for $k$ odd.

The argument above can easily be adapted to the other cases.

(d) Desuspending Whitehead products. In the metastable range, we may use the $J$-homomorphism to translate desuspension problems for certain Whitehead products into sectioning problems for certain vector bundles related to the tangent bundle of the sphere. In particular, our method may be used to give an easy proof of the following theorem due to Barratt and Mahowald.

THEOREM (8.7). Let $\mu_{8 r+1} \in \pi_{8 r+1}^{s}$ be a stable homotopy element with nonzero $d$-invariant and let $\iota_{4 k}, \iota_{4 k+1}$ be the identity map on $S^{4 k}, S^{4 k+1}$ respectively. Then, the Whitehead products $\left[\iota_{4 k}, \mu_{8 r+1}\right]$ and $\left[\iota_{4 k+1}, \mu_{8 r+1}\right]$ desuspend only once in the metastable range. 
Proof. Since we are in the metastable range, we need only to show that the bundles $\mu_{8 r+1}^{*}\left(\tau^{4 k}\right)$ and $\mu_{8 r+1}^{*}\left(\tau^{4 k+1}\right)$ admit at most one section. By (8.1), $\delta_{\Lambda} \mu_{8 r+1}^{*}\left(\tau^{4 k+1}\right)$ and $\delta_{\Lambda}^{+} \mu_{8 r+1}^{*}\left(\tau^{4 k}\right)$ are nonzero for $\Lambda=R$ or $H$ depending on the parity of $k$. If we suppose that $\mu_{8 r+1}^{*}\left(\tau^{4 k+1}\right)$ or $\mu_{8 r+1}^{*}\left(\tau^{4 k}\right)$ admits two sections, a simple computation using the formulae of Theorem (4.2) leads immediately to a contradiction.

(e) Category of a vector bundle. Recall [12] that the category of a vector bundle $\xi$, denoted vecat $(\xi)$, is defined to be the infimum of the cardinalities of the coverings of $X$ by open sets over which $\xi$ is trivial. The following proposition is an easy consequence of the observations of $\S 9$ of [12].

Proposition (8.8). Let $\Lambda=R$ or $C$. Then, the nilpotency index of $\delta_{\Lambda}(\xi)\left(\delta_{\Lambda}^{ \pm}(\xi)\right.$ if applicable) in $K_{\Lambda}(X)$ is a lower bound for vecat $(\xi)$.

For example, let $V_{n, k}$ denote the Stiefel manifold of $k$-frames in $R^{n}$ and let $\eta_{n, k}$ denote the real $(n-k)$-dimensional vector bundle over $V_{n, k}$ associated with the principal fibration $\operatorname{Spin}(n-k) \rightarrow \operatorname{Spin}(n) \rightarrow V_{n, k}$. Let $\eta_{n, k}^{\prime}$ be the restriction of $\eta_{n, k}$ to the truncated projective space $R P^{n-1} / R P^{n-k-1} \subset V_{n, k}$. Proposition (8.8) can be used to show that if $k+n-1$ is even, then vecat $\left(\eta_{n, k}\right) \geqslant s$ where $s$ is the largest odd number smaller than $1+k /(n-k)$. (It should be noticed that, since $\eta_{n, k}$ is stably trivial, usual methods of finding a lower bound for vecat $\left(\eta_{n, k}\right)$ do not apply easily.)

\section{REFERENCES}

1. J. F. Adams, On the groups $J(X)$. II, Topology 3 (1965), 137-172.

2. __ Lectures on Lie groups, Benjamin, New York, 1969.

3. J. Allard, Contribution to the theory of stable trivial vector bundles, Ph.D. Thesis, University of British Columbia, 1977.

4. Sums of stably trivial vector bundles, Math. Proc. Cambridge Philos. Soc. 87 (1980), 97-107.

5. M. F. Atiyah, R. Bott and A. Shapiro, Clifford modules, Topology 3 (1964), 3-38.

6. A. P. Bahri, Problems in algebraic topology, D.Phil. Thesis, Oxford, 1980.

7. A. J. Berrick, Consequences of the Kahn-Priddy Theorem in homotopy and geometry, preprint, Imperial College, London, 1978.

8. R. Bott, Lectures on $K(X)$, Benjamin, New York, 1969.

9. D. Husemoller, Fiber bundles, 2nd ed., Springer-Verlag, New York, 1975.

10. I. M. James, The topology of Stiefel manifolds, London Math. Soc. Lecture Note Series, no. 24, Cambridge Univ. Press, New York, 1976.

11. On category, in the sense of Lusternik-Schnirelmann, Topology 17 (1978), 331-348.

12. M. A. Kervaire, Some nonstable homotopy groups of Lie groups, Illinois J. Math. 4 (1960), 161-169.

13. J. Mukai, An application of the Kahn-Priddy Theorem, J. London Math. Soc. 15 (1977), 183-187.

14. F. Sigrist and U. Suter, Eine Anwendung der K-Theorie in der Theorie der H-Räume, Comment. Math. Helv. 47 (1972), 36-52.

Department of Mathematics, Universite de Moncton, Moncton, New Brunswick, Canada

Department of Mathematics, Rutgers University, New Brunswick, New Jersey 08903 